\title{
Message Optimization based on Scheduling Model for FlexRay Static Segment Application
}

\author{
Xiang-Qi Kong, Yi-Hu Xu, and Yi-Nan X.
}

\begin{abstract}
FlexRay is a high-speed communication protocol with high flexibility and reliability. To identify message optimization of FlexRay static segment, we employed a message optimization scheme based on scheduling model consisting of three steps. First, in order to improve the efficiency of message transmission, a signal packing scheme based on grouping and arrangement method is proposed. Then, to minimize the number of Frame ID (FID) in a FlexRay system, a message scheduling policy with a static slot can be reused by multiple frames. Finally, on the basis of previous steps, scheduling model of static messages frames is described. At the end of this paper, a vehicle chassis system based on FlexRay network is designed. This indicates that the scheduling model can reduce the FID number by $44 \%$, cut down the busload of static by $0.3 \%$, and increase the bandwidth utilization by $12 \%$.
\end{abstract}

Index Terms-FlexRay, FID multiplexing, static segment, signal packing, scheduling model.

\section{INTRODUCTION}

FlexRay is the new standard of network communication system which provides a high speed serial communication, time triggered bus and fault tolerant communication between electronic devices for automotive applications. FlexRay supports a time-triggered scheme and an optional event-triggered scheme. The upper bound of the data rate is $10 \mathrm{Mbps}$ and it provides two channels for redundancy [1]. In 2006, FlexRay communication system was first applied to the electronically controlled dampers of BMW X5 series. FlexRay communication system is applied to vehicle chassis network of BMW 7series, X5, X6, Mercedes Benz S-Class, Audi A8 and so on. Because of expensive cost and relatively complicated communication protocol of FlexRay communication network, it has not been applied popularly in the vehicle communication network system; only to part of the network system of some luxury vehicles.

FlexRay network message scheduling is similar to multi-core parallel scheduling problem of computer system, but has more complex constraints which make it harder to achieve the schedule. One mathematic model of network efficiency optimization is proposed with uncertainty during transmission [2-7]. In order to improve the reliability of frame, a recent study proposes a frame packing method which can calculate frame re-transmitting time. This method leads to bandwidth waste[8-9]. Another study provides the

Manuscript received September 5, 2016; revised December 1, 2016. This work was supported by the National Natural Science Foundation of China (61361003).

The authors are with the College of Engineering of Yanbian University, Yanji, 133002, China (e-mail: 2016050270@ybu.edu.cn, xuyh@ybu.edu.cn, ynxu@ybu.edu.cn). concept of virtual event-driven communication layer and scheduling scheme based on priority which increases the bandwidth efficiency [10], [11]. The disadvantage of this concept is that it overlooks the change of system message period. The slot assignments method based on response time is proposed, but it does not consider the bandwidth utilization. Frame packing and message scheduling is proposed, however it does not consider the FlexRay communication cycle and hard to be applied in real FlexRay communication systems [12]-[14].

The paper provides one message scheduling module for the FlexRay static segment message optimization. The proposed module can get the scheduling list of each node in the network, which improves the bandwidth utilization and decreases the number of FID.

\section{FlexRay Communication Protocol}

The message transmission method of FlexRay is the communication cycle as shown in Fig1. The communication cycle of FlexRay is 0-63 cycles, and 64 cycles which compose one super-cycle [15], [16]. Every communication cycle is made up with the static segment, dynamic segment, symbol window, and network idle time. The static segment is composed of many static slots transmit periodic messages using TDMA and its cycle is an integral multiple of the communication cycle. Each node in the static segment has a scheduling list which contains the transmission periods and slots. The messages are transmitted to their own slot and one slot has one special FID.

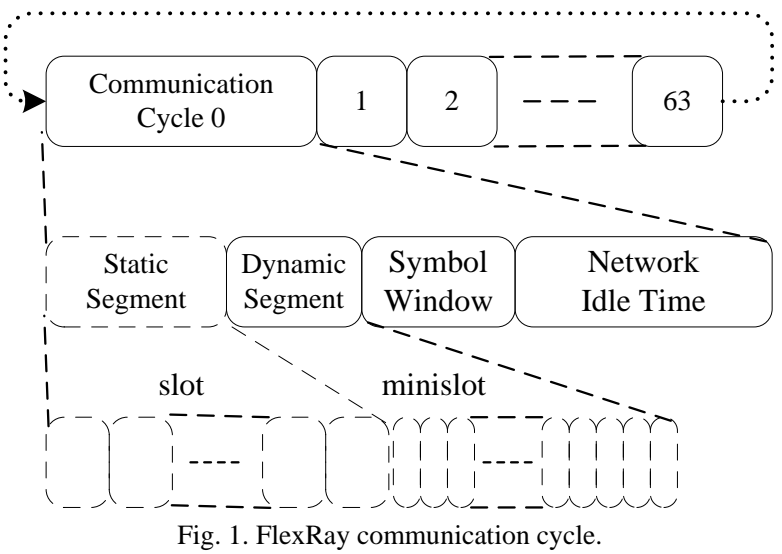

The message frame is the essential unit in FlexRay network as shown in Fig. 2. The message frame is composed with a header segment, payload segment and trailer segment. The length of payload segment containing transmission messages is $0-254$ bytes. The first processing to message 
frame is encoded before transmission. The transmission start sequence occupies 3-15 bits and the frame start sequence occupies 2 bits. BSS (Byte Start Sequence) is added to each byte of message frame.

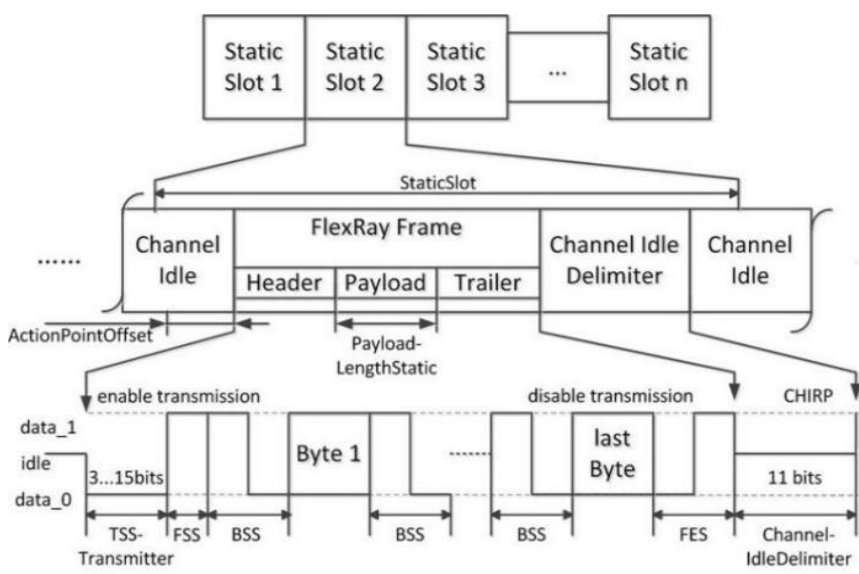

Fig. 2. FlexRay message frame format.

It is assumed that the packed message frame set is $M=\left\{m_{1}, m_{2}, m_{3} \ldots m_{K}\right\}$, in which message $m_{j}(j=1,2,3 \ldots, K)$ is characterized by $\left\{e_{j}, p_{j}, d_{j}, w_{j}\right\}, e_{j}$ is the send node, $p_{j}$ is the period, $d_{j}$ is the deadline, $w_{j}$ is the message length in the size of bit. According to the FlexRay protocol, the message frame size can be as follows:

$$
L_{F L}(j)=T S S+F S S+80(\text { bits })+1.25 w_{j}+F E S
$$

where $L_{F L}$ is the message frame size and 80 bits is the sum number of the frame header and the frame trailer.

Therefore, the static slot length can be calculated by:

$$
\left\{\begin{array}{l}
L_{S T}(j)=2 \times A P O+\operatorname{ceil}(A \times B) \\
A=\left(L_{S T}(j)+C I D\right) \times t_{b i t}+M i n P D+M a x P D \\
B=t_{M T} \times(1-C D M)^{-1}
\end{array}\right.
$$

where $L_{S T}$ is the length of static slot in the size of macrotick (MT), ceil is the top integral function and $t_{b i t}$ is the nominal bit time. The Action Point Offset (APO) is 1 63 MT, Channel Idle Delimiter (CID) is 11 bits, Min Propagation Delay (MinPD) is $0 \sim 2.5 \mu \mathrm{s}$, Max Propagation Delay (MaxPD) is $0 \sim 2.5 \mu \mathrm{s} t_{M T}$ and is the duration of the cluster wide nominal macrotick expressed in $\mu \mathrm{s}$. The Clock Deviation Max (CDM) is 0.0015 .

It is also assumed that there are E nodes in FlexRay bus, the FID(FID) number of each node is denoted as $e_{F I D}(i), i=1,2 \ldots E$, so all the FID number of E nodes is AID, the AID function is as follows:

$$
A I D=\sum_{i=1}^{E} e_{F I D}(i)
$$

Bandwidth utilization represents how much of the static slot is used for transmitting signal data which is determined by the longest message. In this paper, it is defined by:

$$
U=\frac{\sum_{j=1}^{K} W_{j}}{K \max \left(w_{j,} j=1,2, \ldots, K\right)}
$$

Hence, the purpose of the static message scheduling is to minimize the FID number and maximize the bandwidth utilization on the condition that it satisfies the message sending effectively.

\section{SignAl PACKING}

Messages are transmitted as message frame in vehicle communication network. The message frame contains messages and fixed protocol payload which contains this message frame transmission data. As the signals is packed as message frame, many static slots are free, which can be used by the following ECUs. Signal packing scheme improves the bandwidth using efficiency.

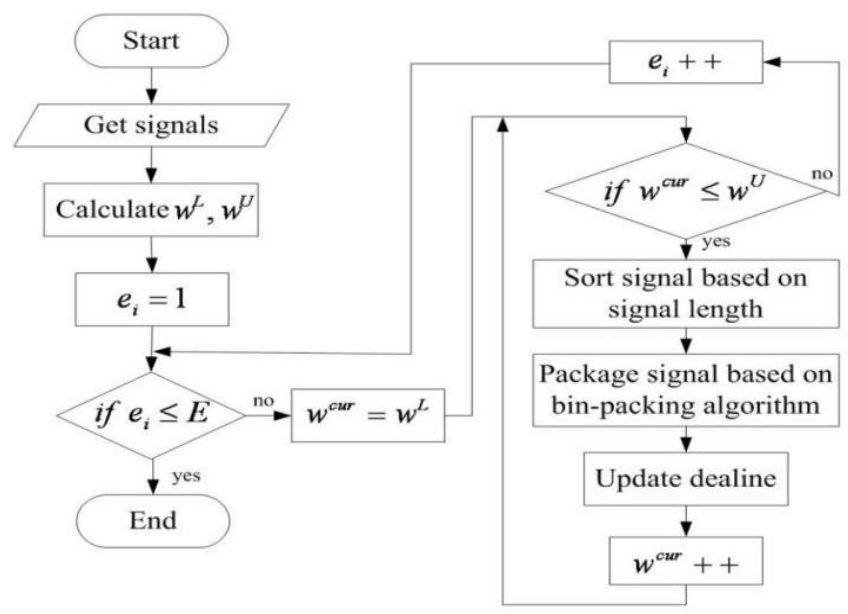

Fig. 3. Frame packing flow chart

The signals which need to be transmitted are $S=\left\{s_{1}, s_{2}, \ldots s_{n}\right\}$, where signal $i$ can be represented as $\left\{e_{j}, p_{j}, d_{j}, b_{j}\right\}, e_{j}$ is the send node, $p_{j}$ is the period, $d_{j}$ is the deadline and $b_{j}$ is the signal length in the size of bit. Signal packing is the process of assembling signal set $S=\left\{s_{1}, s_{2}, \ldots s_{n}\right\}$ into message set $M=\left\{m_{1}, m_{2}, \ldots m_{k}\right\}$. Only signals that have the same send node and period can be packed into one message. A binary variable $x_{i, j}$ is imported to mark whether signal $s_{i}$ is packed into message $m_{j}$ or not, $x_{i, j}=1$ means that the signal is packed into message $m_{j}$, otherwise $x_{i, j}=0$. Each signal has to be packed into only one message as equation (6).

$$
\begin{gathered}
x_{i, j}=\left\{\begin{array}{l}
1, e_{i}=e_{j \text { and }} p_{i}=p_{j} \\
0, \text { otherwise }
\end{array}\right. \\
\sum_{j=1}^{K} x_{i, j}=1, i=1,2, \ldots, S
\end{gathered}
$$


The length of the packed message must be shorter than the length of the static slot. The deadline of the packed message cannot be earlier than the message deadline.

Let the best slot length of the static segment be ,then,

$$
\begin{aligned}
& \sum_{i=1}^{K} x_{i, j} b_{i} \leq w_{b e s t} \\
& d_{j}=\min \left\{d_{i}, d_{j}\right\}
\end{aligned}
$$

In order to decrease the packing time, $w^{L}$ and $w^{U}$ are applied which are the boundaries of $w_{\text {best }}$.

$$
w^{L} \leq w_{\text {best }} \leq w^{U}
$$

The value of $w_{\text {best }}$ is influenced by the messages. If the number of signal and message are equal, it means that one message has only one signal. We can get the greatest value of signal $w^{L}$.

$$
w^{L}=\max b_{i}, i=1,2, \ldots, S
$$

When the number of message and node are equal, which means the signals of one node are packed into one message frame, the value of $w^{U}$ can represented by the length of node signals:

$$
w^{U}=\max _{\{1 \leq k \leq E\}} \sum_{e_{i}=k} b_{i}
$$

The signal packing flow is shown in Fig.3. Firstly, $w^{L}, w^{U}$, and $w_{\text {best }}$ are calculated according to the signal information. Then the signals are divided into different groups and packed using the proposed packing algorithm. In the end, we can get the bandwidth utilization and $w_{\text {best }}$.

\section{FID REUSING}

It is the FID with respect to the frame number that determines the slot of transmitting data frame. Each data frame is assigned with one FID, and one FID can be used only once in each communication cycle. The range of FID is 1 2047. With special FID, the slots of data transmission and the start time is determined. In order to improve the flexibility of static segment scheduling list, the scheduling list can be designed with the least slots, which leaves many free slots for new nodes. Hence, the reusing of FID is important for message scheduling.

After signal packing, we can get the best length of static slot, the number of transmitting message of each node and the periods of each message. The message FID reusing is designed on the basis of signal packing and the reusing scheme minimizes the number of FID in static segment. The messages from the same node can use one FID, and the messages with the greatest common divisor can be transmitted in one slot.

Let $N_{(m, n)}$ be messages number of messages with period $m$ and period $n$ one slot. The message with period 4 can be represented with $1 / 2$ period 2 message, which respect to $1 / 2 \cdot N(2,4)$. The message with period 6 can be represented as $1 / 2 \cdot N(3,6)$ or $1 / 3 \cdot N(2,6)$.

Assuming that node $e(1 \leq e \leq E)$ sends kinds of different periodic messages, the period of each kind message is $T_{i}^{e}\left(1 \leq i \leq n_{e}\right)$. So we define $T^{e}=\left\{T_{1}^{e}, T_{2}^{e}, \ldots, T_{n_{e}}^{e}\right\}$ as the set of different periods of node $e$, the message number of period $T_{i}^{e}$ is $n_{i}^{e}$, then the prime factors of the set $T^{e}$ is denoted as set $Z^{e}=\left\{f_{1}^{e}, f_{2}^{e}, \ldots f_{k^{e}}^{e}\right\}$. Then every period can be expressed as the product of some or all elements in $Z^{e}=\left\{f_{1}^{e}, f_{2}^{e}, \ldots f_{k^{e}}^{e}\right\}$. The number of slots in which send messages of period $f_{k}^{e}$ or messages whose period can be expressed by $f_{k}^{e}$ can be formalized as follows:

$$
\begin{array}{r}
T\left(f_{k}^{e}\right)=\frac{1}{f_{k}^{e}} \sum_{m=1} \operatorname{ceil}\left(\frac{1}{m} N\left(f_{k}^{e}, m f_{k}^{e}\right),\right. \\
m f_{k}^{e} \in T^{e}, m \in N^{*}
\end{array}
$$

The number of slots needed by node $e$ is as follows:

$$
\begin{aligned}
& e_{F I D}(e)=n_{1}^{e}+\sum_{k=1}^{k^{e}} T\left(T\left(f_{k}^{e}\right)\right. \\
& \left\{\begin{array}{c}
\sum_{f_{k}^{e} \in Z^{e}} N\left(f_{k}^{e}, T_{i}^{e}\right)=n_{i}^{e} \\
N\left(f_{1}^{e}, T_{i}^{e}\right) \geq 0 \\
N\left(f_{2}^{e}, T_{i}^{e}\right) \geq 0 \\
\vdots \\
\vdots \\
N\left(f_{k^{e}}^{e}, T_{i}^{e}\right) \geq 0
\end{array}\right.
\end{aligned}
$$

Where, equation (13) is an objective function and equation (14) is a constraint. Considering the problem formulation, the FID reuse problem can be translated into nonlinear integer programming. Then the number of slots for node $e$ can be obtained, and so on, for other nodes.

\section{The design of Static Segment Message Scheduling}

The nodes in FlexRay network have their own buffer area which can store the messages waiting for transmission. The assignments of message transmission order are on the basis of scheduling list including transmission period and FID in the scheduling list. In the bus communication protocol, the message can be transmitted after encoding according to the scheduling list. The design of scheduling list influences the whole communication network. Each message $m_{j}$ includes:

$$
S D_{j}=\left\{b s_{j}, s r_{j}, n s_{j}, b c_{j}, c r_{j}, n c_{j}\right\}
$$

Each message is transmitted on the base slot $b s_{j}$ and base cycle $b c_{j}$, it happens once after $s r_{j}$ slots in one cycle and repeats after $c r_{j}$ cycles. 
$n s_{j}$ is the slot number for transmitting message $m_{j}$ in one communication cycle, $n c_{j}$ is the cycle number of $m_{j}$ in one super-cycle. When a message is only transmitted once in one cycle, namely $n s_{j}=1$, then $s r_{j}=0$.

In order to determine the value of $b c_{j}$ and $b s_{j}, 2 \mathrm{D}$ vector $v$ is defined. The abscissa of the vector is the static slots ID, the greatest value is $N_{S T S}$; the ordinate is communication cycle and the greatest value is 63 . The message transmission start position $\left(b s_{j}, b c_{j}\right)$ of message $m_{j}$ is called the start point which is very important for the communication network.

All the possible start points of message $m_{j}$ is one set $v_{j}$. Where $v_{j} \in V$ and $\left(b s_{j}, b c_{j}\right) \in v_{j}$. The reference point of Message $m_{j}$ must be in the interval of two communications because of the message periodical transmission. The range of $b s_{j}$ is $\left[1, s r_{j}\right]$ and the range of $b c_{j}$ is $\left[0, c r_{j}-1\right]$. Hence, the range of start points of message $m_{j}$ is $v_{j}=\left(\left[1, s r_{j}\right],\left[0, c r_{j}-1\right]\right)$.

The message is worst response time depends on the time from message generating to message receiving. In order to transmit the message in time, the worst response time cannot be later than the message period and it must be earlier than the message deadline. The worst response time of static segment message is:

$$
W C R T_{j}=\left(c r_{j}-1\right) T_{c}+s r_{j} \times T_{s l o t}
$$

where, $T_{c}$ is communication period, $T_{\text {slot }}$ is the length of static slot. The constraints $c r_{j}$ of and $s r_{j}$ is:

$$
\begin{gathered}
W C R T_{j} \leq d_{j} \\
W C R T_{j} \leq p_{j} \\
c r_{j}=\left\{2^{n} \mid n \in[0,6], n \in Z\right\} \\
s r_{j}=\left\{2^{n} \mid n \in\left[0, \log _{2} N_{S T S}\right], n \in Z\right\}
\end{gathered}
$$

The message scheduling is designed according to the value of $s r_{j}$, because of the high demands of communication time. The message with smaller $c r_{j}$ has higher scheduling priority when two messages have the same $s r_{j}$.After the scheduling of message $m_{j}$, the reference point occupation of the next message $m_{j+1}$ does not include the slot of message $m_{j}$.

\section{EXPERIMENTS RESULTS}

In order to evaluate the efficiency of the proposed algorithm, it is compared with the rate monotonic scheduling algorithm(RMS). Simulation parameter is shown in table 1, where signal cycle is integer times of the communication cycle, signal deadline is signal cycle.

\begin{tabular}{cc} 
TABLE I: PARAMETER SETTING OF FLEXRAY NETWORK \\
\hline parameter & value \\
\hline Communication rate & $10 \mathrm{Mbit} / \mathrm{s}$ \\
Communication cycle & $5 \mathrm{~ms}$ \\
Node number & 10 \\
Signal number & $10-300$ \\
\hline
\end{tabular}

On the basis of scheduling performance evaluation index, two kinds of scheduling algorithm are simulated, results are shown in figure 4 and figure 5 . The bandwidth utilization of two scheduling algorithms are described in figure 4 . The proposed algorithm exceeds the rate monotonic scheduling algorithm in bandwidth utilization, and with the increase of signal number the proposed algorithm is significantly better than the rate monotonic scheduling algorithm. The FID number of two algorithms are shown in figure 5. With the increase of message number, the FID number of the rate monotonic scheduling algorithm growth linearly, however the FID number of the proposed algorithm increases slightly. So the proposed algorithm is superior to the rate monotonic scheduling algorithm in the scheduling problem of the static segment.

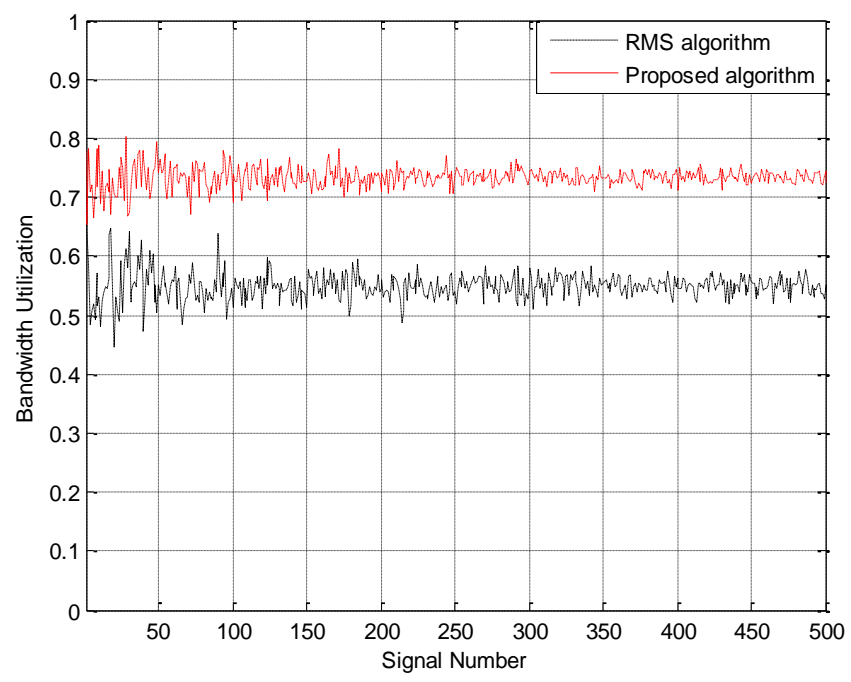

Fig. 4. Bandwidth utilization.

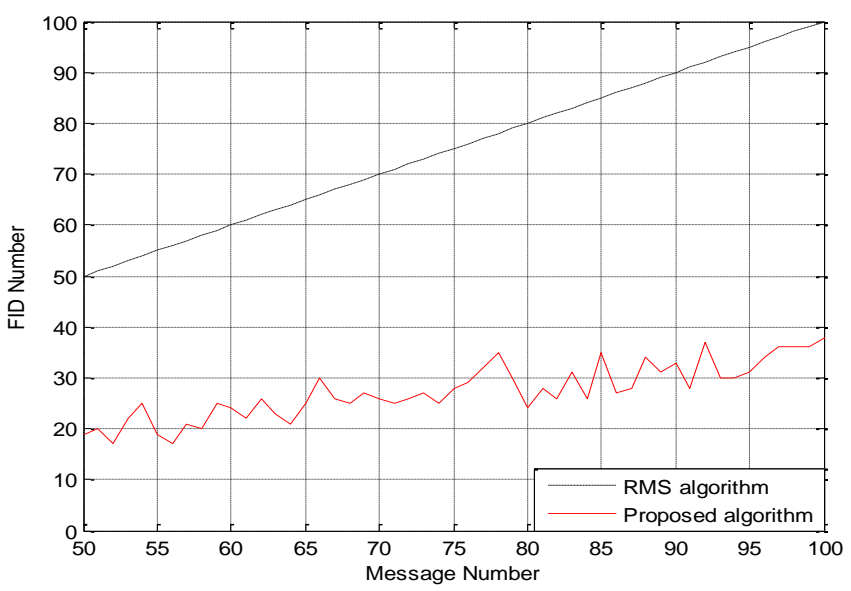

Fig. 5. The required number of FIDs.

TABLE II: FLEXRAY NETWORK PARAMETER VALUES

\begin{tabular}{ccc}
\hline parameter & value & unit \\
\hline TSS & 9 & Bits/frame \\
FSS & 1 & Bit/frame \\
BSS & 2 & Bits/payload byte \\
MinPD & 1 & us \\
$t_{b i t}$ & 0.1 & us \\
CID & 11 & Bits/frame \\
APO & 6 & MT/frame \\
FES & 2 & Bits/frame \\
MaxPD & 1.5 & us \\
$t_{M T}$ & 1 & us \\
\hline
\end{tabular}

The vehicle chassis communication system is applied in 
the simulation experiments. The communication cycle is $5 \mathrm{~ms}$ and the static segment length is $3 \mathrm{~ms}$. The other parameters are shown in Table II. There are 5 nodes in the bus with 20 messages. The distributions are shown in Table III.

\begin{tabular}{ccccc}
\multicolumn{5}{c}{ TABLE III: FLEXRAY NETWORK SIGNAL VALUES } \\
\hline signal & $e_{i}$ & $p_{i} / \mathrm{ms}$ & $d_{i} / \mathrm{ms}$ & $b_{i} / \mathrm{bit}$ \\
\hline$s_{1}$ & 5 & 10 & 10 & 26 \\
$s_{2}$ & 1 & 5 & 5 & 2 \\
$s_{3}$ & 4 & 20 & 20 & 2 \\
$s_{4}$ & 4 & 20 & 20 & 8 \\
$s_{5}$ & 5 & 20 & 20 & 16 \\
$s_{6}$ & 1 & 5 & 5 & 16 \\
$s_{7}$ & 3 & 10 & 10 & 8 \\
$s_{8}$ & 1 & 5 & 5 & 4 \\
$s_{9}$ & 3 & 40 & 40 & 64 \\
$s_{10}$ & 4 & 40 & 40 & 16 \\
$s_{11}$ & 4 & 20 & 20 & 32 \\
$s_{12}$ & 1 & 5 & 5 & 32 \\
$s_{13}$ & 2 & 5 & 5 & 16 \\
$s_{14}$ & 3 & 10 & 10 & 8 \\
$s_{15}$ & 4 & 40 & 40 & 32 \\
$s_{16}$ & 3 & 10 & 10 & 32 \\
$s_{17}$ & 3 & 10 & 10 & 16 \\
$s_{18}$ & 5 & 20 & 20 & 8 \\
$s_{19}$ & 4 & 40 & 40 & 16 \\
$s_{20}$ & 2 & 5 & 5 & 32 \\
\hline & & & &
\end{tabular}

The constraints of the messages packing are message node and periods. This means that messages with the same nodes and period are packed into one group. The packing results are shown in Table IV.

TABLE IV: SIGNAL PACKING

\begin{tabular}{cccccc}
\hline message & $e_{i}$ & $p_{i} / \mathrm{ms}$ & $d_{i} / \mathrm{ms}$ & $\mathrm{w}_{\mathrm{i}} / \mathrm{bit}$ & Signal \\
\hline$m_{1}$ & 1 & 5 & 5 & 54 & $s_{2} s_{6} s_{8} s_{12}$ \\
$m_{2}$ & 2 & 5 & 5 & 48 & $s_{13} s_{20}$ \\
$m_{3}$ & 3 & 10 & 10 & 64 & $s_{7} s_{14} s_{16} s_{17}$ \\
$m_{4}$ & 3 & 40 & 40 & 64 & $s_{9}$ \\
$m_{5}$ & 4 & 40 & 40 & 16 & $s_{10}$ \\
$m_{6}$ & 4 & 20 & 20 & 42 & $s_{3} s_{4} s_{11}$ \\
$m_{7}$ & 4 & 40 & 40 & 48 & $s_{15} s_{19}$ \\
$m_{8}$ & 5 & 10 & 10 & 26 & $s_{1}$ \\
$m_{9}$ & 5 & 20 & 20 & 24 & $s_{5} s_{18}$ \\
\hline
\end{tabular}

According to the FID reusing algorithm, the number of FID is decreased which is shown in Table 5.The greatest common divisor of $m_{3}$ and $m_{4}$ is not 1 , so the two messages can use the same slot. Then, the messages $m_{5}, m_{6}, m_{7}$ of node 4 can use one slot and the messages $m_{8}$ and $m_{9}$ of node 5 can also use the same slot.

TABLE V: FID MULTIPLEXING

\begin{tabular}{|c|c|c|c|c|c|}
\hline message & $e_{i}$ & $p_{i} / \mathrm{ms}$ & $d_{i} / \mathrm{ms}$ & $\mathrm{w}_{\mathrm{i}} / \mathrm{bit}$ & Signal \\
\hline$m_{1}$ & 1 & 5 & 5 & 54 & $m_{1}$ \\
\hline$m_{2}^{\prime}$ & 2 & 5 & 5 & 48 & $m_{2}$ \\
\hline$m_{3}$ & 3 & 10 & 10 & 64 & $m_{3} m_{4}$ \\
\hline
\end{tabular}

\begin{tabular}{cccccc}
\hline$m_{4}^{\prime}$ & 4 & 20 & 20 & 42 & $m_{5} m_{6} m_{7}$ \\
$m_{5}^{\prime}$ & 4 & 40 & 40 & 48 & $m_{8} m_{9}$ \\
\hline
\end{tabular}

The message scheduling list is shown in Table 6. Where, $b s_{j}$ is the message essential slot, $b c_{j}$ is the message transmission essential period. And 5 messages are transmitted at the start.

\begin{tabular}{ccccccc}
\multicolumn{7}{c}{ TABLE VI: MESSAGE SCHEDULING TABLE } \\
\hline message & $b s_{j}$ & $s r_{j}$ & $n s_{j}$ & $b c_{j}$ & $c r_{j}$ & $n c_{j}$ \\
\hline$m_{1}^{\prime}$ & 1 & 0 & 1 & 0 & 1 & 64 \\
$m^{\prime}{ }_{2}$ & 2 & 0 & 1 & 0 & 1 & 64 \\
$m_{3}^{\prime}$ & 3 & 0 & 1 & 0 & 2 & 32 \\
$m^{\prime}{ }_{4}$ & 5 & 0 & 1 & 0 & 4 & 16 \\
$m_{5}^{\prime}$ & 4 & 0 & 1 & 0 & 2 & 32 \\
\hline
\end{tabular}

The experiments are based on Network Designer, FlexRay and CANoe. FlexRay is designed to evaluate the efficiency of the proposed algorithms in the paper. The concrete node parameters are shown in Fig. 6. ECU_1, ECU_2, ECU_3, ECU_4, and ECU_5 are transmission node and ECU_6 is a receiving node. The messages transmitted in each node are shown in Table 3. On the basis of message scheduling with respect to the communication parameters, the database is built by Network Designer. Then, the database is imported into the softwares CANoe and FlexRay.

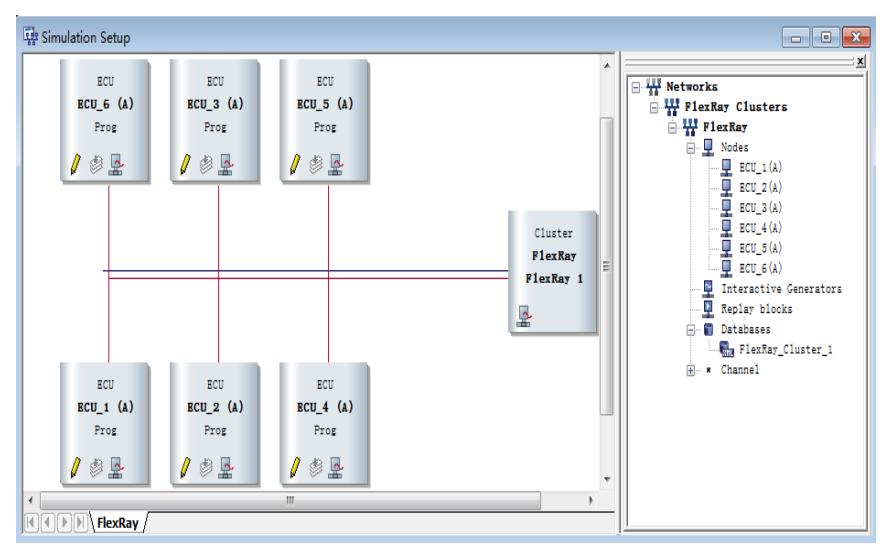

Fig. 6. The node set of FlexRay simulation system.

The communication rate is $10 \mathrm{Mb} / \mathrm{s}$ in the simulation system. The paper simulates two algorithms including FID and FID reusing. The network payload results are shown in Table 6. The FID reusing method decreases the payload of static segment, and increases the bandwidth efficiency. The network gets good extension with less FID.

TABLE VII: NETWORK PERFORMANCE COMPARISON TABLE

\begin{tabular}{lll}
\hline network performance & FID reusing before & FID reusing after \\
\hline Total frames & 775 & 775 \\
Null frames & 0 & 0 \\
Busload & $5.60 \%$ & $5.58 \%$ \\
bandwidth utilization & $67 \%$ & $75 \%$ \\
FID number & 9 & 5 \\
\hline
\end{tabular}

\section{CONCLUSION}

This paper focuses on the research of FlexRay static 
segment scheduling. According to the static segment message transmission feature of FlexRay, the message evaluation criteria are presented including the bandwidth efficiency, and the number of FID. On the basis of message optimization, the message scheduling model is built. The simulation results show that FID number decreases by $44 \%$, the bandwidth improves $12 \%$ and the bus payload decreases by $0.3 \%$. The FlexRay static segment message improves the transmission efficiency.

\section{REFERENCES}

[1] FlexRay, "FlexRay communications system protocol specification," 2010.

[2] K. Park, M. Kang, and B. Kim, "A scheduling algorithm for reducing FlexRay message response time using empty minislots in dynamic segment," in Proc. Digest of Technical Papers International Conference on Consumer Electronics, pp. 307-308, 2010.

[3] A. Darbandi, S. Kwon, and M. K. Kim, "Scheduling of time triggered messages in static segment of FlexRay," International Journal of Software Engineering and Its Applications, vol. 8, no. 6, pp. 195-208, 2014.

[4] Y. Hua, X. Liu, W. B. He, and D. Feng, "Design and implementation of holistic scheduling and efficient storage for FlexRay," IEEE Transactions on Parallel and Distributed Systems, vol. 25, no. 10, pp. 2529-2539, 2014.

[5] B. Seo and D. Lee, "Determining the length of static message for efficient use of FlexRay network," SICE Annual Conference, pp. 563-566, 2010.

[6] R. Zhao, G. H. Qin, and J. Q. Liu, "A rectangle bin packing optimization approach to the signal scheduling problem in the FlexRay static segment," Frontiers of Information Technology Electronic Engineering, vol. 17, no. 4, pp. 375-388, 2016.

[7] G. Han, H. Zeng, Y. Li et al., "Security-aware flexray scheduling engine," Design Automation and Test in Europe, pp. 1-4, 2014.

[8] B. Tanasa, U. Dutta Bordoloi et al., "Reliability-aware frame packing for the static segment of flexray," in Proc. the Ninth ACM International Conference on Embedded Software, pp. 175-184, 2011.

[9] M. Kang, K. Park, and M. K. Jeong, "Frame packing for minimizing the bandwidth consumption of the FlexRay static segment," IEEE Transactions on Industrial Electronics, vol. 60, No. 9, pp. 4001-4008, 2013.

[10] P. Mundhenk, F. Sagstetter, and S. Steinhorst, "Policy-based message scheduling using flexray," in Proc. the 2014 International Conference on Hardware/Software Codesign and System Synthesis, pp. 1-10, 2014.

[11] H. B. Zeng, M. Natale, A. Ghosal, and A. Sangiovanni-Vincentelli, "Schedule optimization of time-triggered systems communicating over the FlexRay static segment," IEEE Transactions on Industrial Informatics, vol. 7, no. 1, pp. 1-17, 2011.

[12] R. Lange and F. Vasques, "Guaranteeing real-time message deadlines in the flexray static segment using an on-line scheduling approach," in Proc. the 9th IEEE International Workshop on Factory Communication Systems, pp. 301-310, 2012.

[13] R. Lange, F. Vasques et al., "A scheme for slot allocation of the FlexRay Static Segment based on response time analysis," Computer Communications, pp. 65-76, 2015.

[14] K. Schmidt and E. G. Schmidt, "Message scheduling for the flexray protocol: the static segment," IEEE Transactions on Vehicular Technology, vol. 58, no. 5, pp. 2170-2179, 2009.

[15] Y. N. Xu, Y. E. Kim, K. J. Cho, J. G. Chung, and M. S. Lim, "Implementation of FlexRay protocol with an automotive application," IEEE International SoC Design Conference, pp. 25-28, 2008.

[16] Y. N. Xu, Y. E. Kim, K. J. Cho, J. G. Chung, and X. M. Huang, "Implementation of FlexRay CC and BG protocols with application to a robot system," Journal of Computer Aided Engineering and Technology, vol. 2, no. 1, pp. 112-122, 2010.

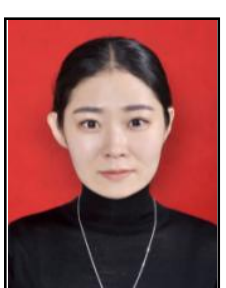

Xiang-Qi Kong was born at Jilin Province of China. She completed bachelor degree in the field of electronics and telecommunication, Yanbian University, China, in 2016

She is still pursuing her Master Degree in the school of Yanbian University, Yanji, China.

Her research interests include the In-vehicle FlexRay network.

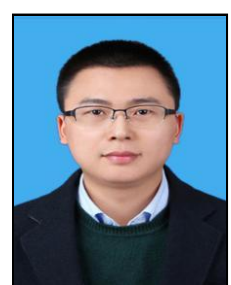

Yi-Hu Xu was born at Jilin Province of China. He received the Ph.D. degree in electronics engineering from the Chonbuk National University, Korea, in 2014.

$\mathrm{He}$ is a lecturer of the division of electronic and communication engineering of Yanbian University, Yanji, China.

His research interests include the automobile electronic control and network.

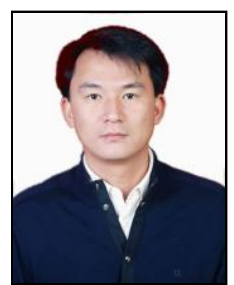

Yi-Nan Xu was born at Jilin Province of China Currently, he is an professor of the division of electronic and communication engineering of Yanbian University, Yanji, China.

$\mathrm{He}$ received the Ph.D. degree in electrical engineering from the Chonbuk National University, Korea, in 2009.

His research interests include the in-vehicle networks and automobile electronic control. 